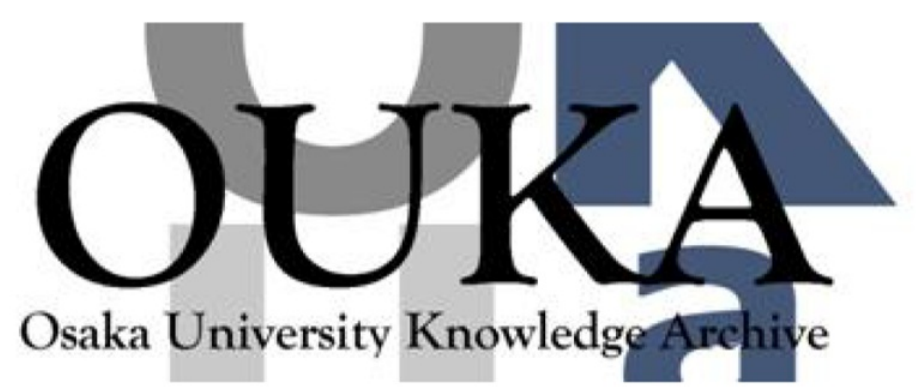

\begin{tabular}{|c|l|}
\hline Title & $\begin{array}{l}\text { Relaxation kinetics of photo induced surface } \\
\text { relief grating on azopolymer films }\end{array}$ \\
\hline Author(s) & $\begin{array}{l}\text { Matsui, Tatsunosuke; Yamamoto, Shin-ichiro; } \\
\text { Ozaki, Masanori et al. }\end{array}$ \\
\hline Citation & $\begin{array}{l}\text { Journal of Applied Physics. 92(12) p. 6959- } \\
\text { p. } 6965\end{array}$ \\
\hline Issue Date & $2002-11-27$ \\
\hline oaire:version & VoR \\
\hline URL & https://hdl. handle. net/11094/75850 \\
\hline rights & \\
\hline Note & \\
\hline
\end{tabular}

Osaka University Knowledge Archive : OUKA

https://ir. Library. osaka-u. ac. jp/

Osaka University 


\section{Relaxation kinetics of photoinduced surface relief grating on azopolymer films}

Cite as: Journal of Applied Physics 92, 6959 (2002); https://doi.org/10.1063/1.1516264

Submitted: 29 May 2002 . Accepted: 28 August 2002 . Published Online: 27 November 2002

Tatsunosuke Matsui, Shin-ichiro Yamamoto, Masanori Ozaki, Katsumi Yoshino, and Francois Kajzar

\section{ARTICLES YOU MAY BE INTERESTED IN}

In-situ atomic force microscopy study of the mechanism of surface relief grating formation in photosensitive polymer films

Journal of Applied Physics 113, 224304 (2013); https://doi.org/10.1063/1.4809640

On the long time holographic grating recording process in azo-polymer

Applied Physics Letters 95, 123302 (2009); https://doi.org/10.1063/1.3232223

Stochastic model for photoinduced surface relief grating formation through molecular transport in polymer films

Applied Physics Letters 93, 153304 (2008); https://doi.org/10.1063/1.2999625

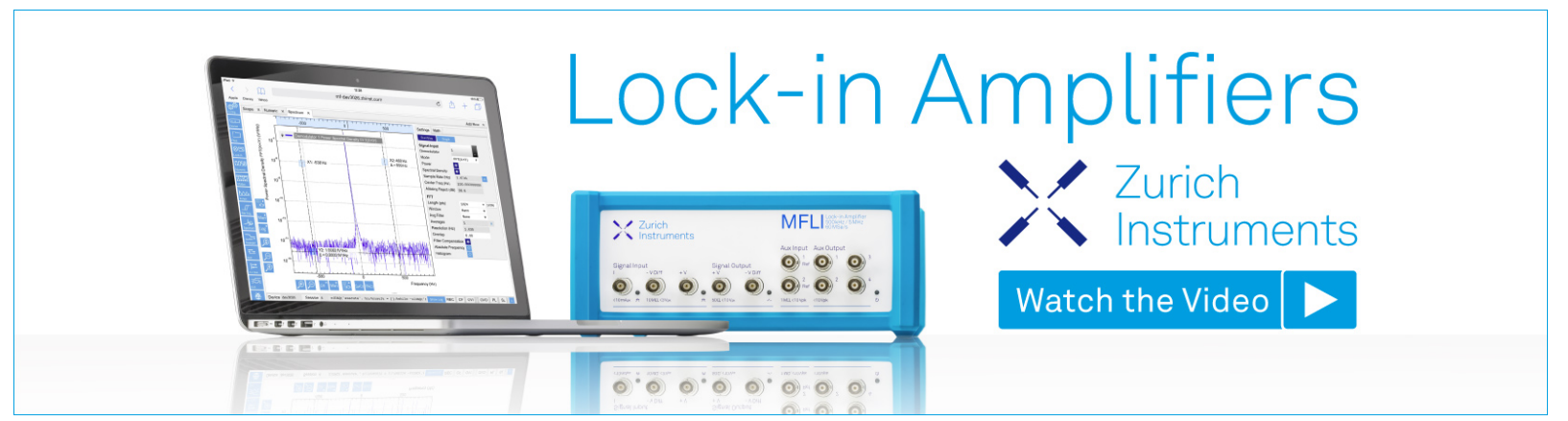




\title{
Relaxation kinetics of photoinduced surface relief grating on azopolymer films
}

\author{
Tatsunosuke Matsui, Shin-ichiro Yamamoto, Masanori Ozaki, ${ }^{a}$ and Katsumi Yoshino \\ Department of Electronic Engineering, Graduate School of Engineering, Osaka University, \\ 2-1 Yamada-Oka, Suita, Osaka 565-0871, Japan \\ Francois Kajzar \\ LPEM/DEIN Centre d'Etudes Nucleaires de Saclay, 91191 Gif/Yvette Cedex, France
}

(Received 29 May 2002; accepted 28 August 2002)

\begin{abstract}
The relaxation kinetics of the photoinduced surface relief grating (SRG) formed on an azopolymer film after stopping laser beam writing has been systematically investigated. In addition to a photoinduced increase of the diffraction efficiency in the SRG, an anomalous further enhancement of the efficiency was observed even without light irradiation, after the recording light beam was switched off. This anomalous relaxation process consists of two components with short and long relaxation times, and strongly depends on the temperature and polarization of the probe beam used for the diffraction measurement. At lower temperatures the anomalous growth is more effective and the fast component is dominant. From the results of the polarization dependence of the diffraction efficiency and optical absorption, it has been manifested that the fast component of the relaxation originates from the cis-trans thermal isomerization of azobenzene in the side chain. The origin of the slow decay is also discussed in terms of the order parameter change of the azomolecules caused by the reorientational relaxation. (C) 2002 American Institute of Physics.
\end{abstract}

[DOI: 10.1063/1.1516264]

\section{INTRODUCTION}

Recently, a holographic recording of a surface relief grating (SRG) on a thin film of an azobenzene-substituted photochromic polymer upon irradiation of two interfering beams from a low-power laser has been discovered. ${ }^{1,2}$ Many studies have been carried out from the viewpoint of fundamental interest and for practical applications such as a grating coupler, ${ }^{3,4}$ an alignment layer of liquid crystal, ${ }^{5}$ a distributed feedback (DFB) laser, ${ }^{6}$ etc. Due to the cyclic trans-cistrans photoisomerization of azobenzene in the side chain, mass transport is induced to form SRG following the distribution of interfered light intensity. For a complete description of the formation mechanism of SRG, both the driving force for the mass transport of the polymer main chain, and the migration process of the polymer should be manifested. Several models to explain the formation mechanism of SRG have been proposed: a radiation force caused by a light intensity gradient, ${ }^{7,8}$ interaction between dipoles of azobenzene moieties (mean-field theory) ${ }^{9}$ as the origin of the driving force, the fluid dynamics model based on the Navier-Stokes equation ${ }^{10,11}$ concerning the formation process, etc. However, the detailed formation mechanism of SRG has not yet been clarified.

From the viewpoint of application of SRG to optical devices, we fabricated the SRG on a composite film of conducting polymer and azopolymer and observed directional photoluminescence affected by the SRG structure. ${ }^{12,13}$ In previous studies, we observed an anomalous increase of dif-

${ }^{a)}$ Electronic mail: ozaki@ele.eng.osaka-u.ac.jp fraction efficiency even without light irradiation after the interfered recording beams were switched off. This phenomenon implies that the mass transport of the polymer main chain and/or azobenzene in the side chain continues through some kind of relaxation process even under the dark condition. Through the understanding of these relaxation mechanisms, it is expected that information about the movement of azobenzene and the polymer main chain can be obtained and the fabrication mechanism of SRG can also be manifested. In this study, the anomalous relaxation dynamics of the SRG formed on the azopolymer film after stopping laser beam writing was investigated under various conditions, such as temperature dependence, and polarization dependence, and the relaxation mechanism of the SRG is discussed.

\section{EXPERIMENT}

\section{A. Material and preparation of thin film}

Figure 1 shows the molecular structure of the polyacrylate containing an azobenzene moiety in the side chain (azopolymer). In this study, every experiment was performed with pure azopolymer that did not contain a conducting polymer, as in Ref. 12. This azopolymer was dissolved in chloroform, spin coated on a glass substrate, and then heated to $90{ }^{\circ} \mathrm{C}$ in vacuum for $12 \mathrm{~h}$ in order to remove residual solvent completely. The thickness of the film was about $1 \mu \mathrm{m}$, which was estimated from the absorbance.

\section{B. Fabrication and observation of SRG}

The SRG was formed using the interferential light irradiation system shown in Fig. 2(a). The recording light source 




FIG. 1. Molecular structure of azopolymer used in this study.

was an $\mathrm{Ar}^{+}$laser (Beamlok2060-4S: Spectra Physics) of 488 $\mathrm{nm}$ wavelength, and the two recording lights were circularly polarized. Based on the following equation, the periodicity $\Lambda$ of SRG can be controlled by changing the angle $2 \theta$ between the two recording laser beams:

$$
\Lambda=\lambda / 2 \sin \theta,
$$

where $\lambda$ is the wavelength of the writing laser light (in this case, $488 \mathrm{~nm}$ ). In this study, the angle $\theta$ was kept at $20^{\circ}$, so that the periodicity of fabricated SRGs was about $700 \mathrm{~nm}$. The recorded SRG profile was observed using an atomic force microscope (AFM), (JSTM-4200A: JEOL).

\section{Evaluation of diffraction efficiency}

In order to monitor the formation of SRG in situ, the intensity of transmitted and diffracted beams of a $\mathrm{He}-\mathrm{Ne}$ laser light $(632.8 \mathrm{~nm})$ was measured by a photodiode and diffraction efficiency was evaluated as shown in Fig. 2(a). A color filter (O-56: TOSHIBA) which cut off light with wavelength shorter than $560 \mathrm{~nm}$, was placed in front of the detector to prevent the detection of the writing $\mathrm{Ar}^{+}$laser light. The polarization of the probe $\mathrm{He}-\mathrm{Ne}$ laser light was perpendicular to the grating vector of SRG. The holographic recording was conducted for $90 \mathrm{~min}$, and after stopping $\mathrm{Ar}^{+}$laser light irradiation, the diffraction efficiency was monitored to evaluate the relaxation dynamics under the dark condition.

As discussed in the following section, the diffraction efficiency depends strongly on the polarization of the probe

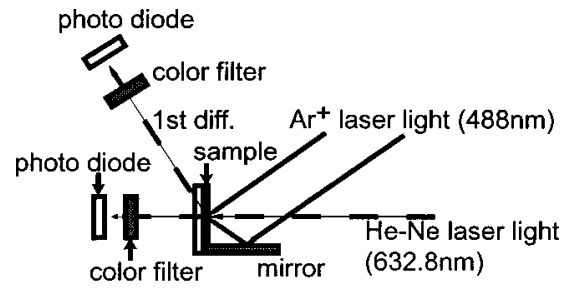

(a)

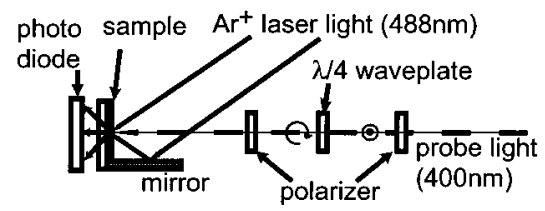

(b)

FIG. 2. (a) Interferential light irradiation system for the holographic formation of SRG, (b) experimental geometry for the measurement of the absorption.

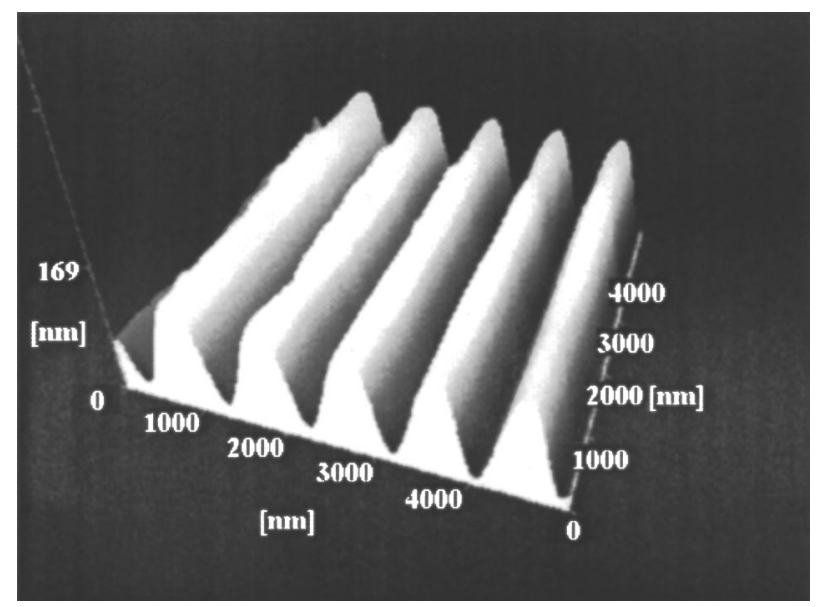

FIG. 3. AFM profile of the formed SRG on the azo-polymer film.

$\mathrm{He}-\mathrm{Ne}$ laser light. In order to investigate that polarization dependence, the diffraction efficiency was also evaluated both by parallel and perpendicularly (to the grating vector) polarized $\mathrm{He}-\mathrm{Ne}$ laser lights.

\section{Measurement of absorption}

In order to monitor the change in the alignment direction of the azochromophore under the dark condition, the change in transmittance of a low-power blue diode laser light of 400 $\mathrm{nm}$ wavelength (LDT-4005: Nichia) was investigated. Azobenzene absorbs light of $400 \mathrm{~nm}$ wavelength. Therefore, by detecting the change of the absorbance, the alignment change of azobenzene can be evaluated. On the other hand, because the absorbance of azobenzene at $400 \mathrm{~nm}$ is small, a photoisomerization effect due to the illumination of blue laser can be neglected. Generally, azobenzene tends to align its optical axis (molecular long axis) perpendicular to the polarization direction of the illuminated light when irradiated with linearly polarized light (Weigert effect), so the intensity of the probe laser light should be low. From this point of view, the laser of $400 \mathrm{~nm}$ wavelength was favorable.

The probe laser beam was passed through a polarizer and a $\lambda / 4$ wave plate in order to make its polarization circular. The polarizer was inserted between the $\lambda / 4$ wave plate and the sample in order to make its polarization linear. By rotating this second polarizer, polarization-dependent absorption change was detected. The setup for absorption measurement is shown in Fig. 2(b). All components of the probe beam, that is, both transmitted light and +1 and -1 diffracted lights, were detected so that the change of absorption alone could be evaluated. In other words, the polarization dependence of the diffraction efficiency could be ruled out.

\section{RESULTS AND DISCUSSION}

Figure 3 shows the AFM profile of the formed SRG on the azopolymer film. From this figure, it was confirmed that the SRG of about $100 \mathrm{~nm}$ in height and $700 \mathrm{~nm}$ in periodicity was recorded. In this case, the dimensionless $Q$ factor of the formed SRG is about 0.5, so that the formed SRG can be 




FIG. 4. Typical irradiation time dependence of diffraction efficiency. The anomalous further enhancement of efficiency can be seen after the recording laser light is switched off.

regarded as a "thin" grating and the diffraction from this SRG should be treated as a Raman-Nath-type diffraction. The $Q$ factor is defined as

$$
Q=2 \pi \lambda d / n \Lambda^{2} .
$$

Here, $\lambda$ is the wavelength of a probe light, $d$ is the thickness of the SRG, $n$ is the refractive index of azopolymer, and $\Lambda$ is the period of SRG. On the other hand, the absorbance of azopolymer at the wavelength of $\mathrm{He}-\mathrm{Ne}$ laser $(632.8 \mathrm{~nm}$ in wavelength) is almost zero so the formed SRG can be regarded as the Raman-Nath-type pure phase grating. ${ }^{14,15}$

Figure 4 shows the diffraction efficiency of the formed SRG as a function of recording time. Just after the start of recording, the grating structure was formed, and the diffraction efficiency gradually increased by sequential light illumination. It should be noted from Fig. 4 that the increase of the diffraction efficiency is observed even after stopping the irradiation of the recording laser. This means that some of the relaxation process occurs under the dark condition. In order to discuss the anomalous relaxation process, the diffraction from both the "surface relief grating" and the "refractive index grating" should be considered. Based on these considerations, possible models explaining the increase of the diffraction efficiency after stopping laser light irradiation can be summarized as follows: (1) change of the relief profile (relief height) itself. This may be attributed to the relaxation of the polymer main chains; (2) Cis-trans thermal isomerization of azobenzene; (3) orientational change of azochromophore in the side chain. This should cause the change in birefringence and absorption. In order to examine which model is suitable for the anomalous relaxation process after stopping the recording laser light irradiation, the following experiments were carried out systematically.

\section{A. Temperature dependence}

In order to investigate the dynamic characteristics of azochromophores in the anomalous relaxation process, the recording of SRG was performed at various temperatures because relaxation dynamics may be affected by thermal conditions. Figure 5(a) shows the irradiation time dependence of the diffraction efficiency at various temperatures $\left(17,37,78\right.$, and $\left.93^{\circ} \mathrm{C}\right)$, and Fig. 5(b) shows the temperature dependence of the final values of the diffraction efficiency at

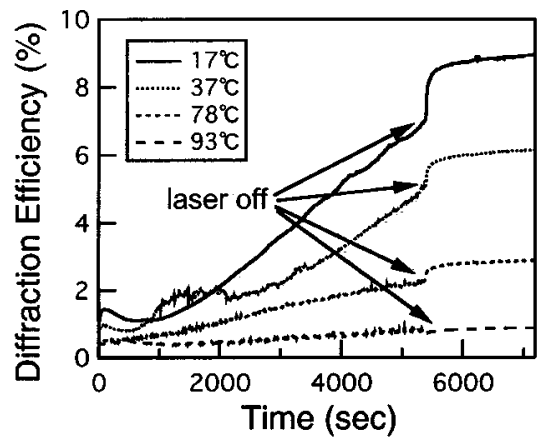

(a)

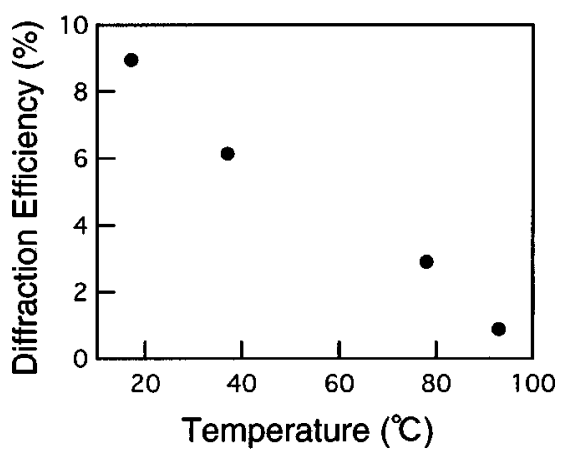

(b)

FIG. 5. (a) Irradiation time dependence of diffraction efficiency at various temperatures $\left(17,37,78\right.$, and $\left.93{ }^{\circ} \mathrm{C}\right)$; (b) Temperature dependence of final value of diffraction efficiency [at $t=7200 \mathrm{~s}$ in Fig. 5 (a)].

$7200 \mathrm{~s}$ in Fig. 5(a). These figures clearly show that the formation of SRG is more effective at lower temperatures.

The migration or reorientation dynamics of the polymer main chains and/or azobenzene in the side chain (first and third models) should depend on the rheological characteristics of azopolymer, such as viscosity. Generally, these characteristics are temperature dependent. On the other hand, if the diffraction efficiency increase originates from a cis-trans thermal isomerization of azobenzene (second model), it should be more effective and the diffraction efficiency will increase faster at higher temperatures because the cis form of azobenzene is unstable thermally and cis-trans thermal isomerization occurs more effectively at higher temperatures. However, the diffraction efficiency is suppresed at higher temperatures as shown in Fig. 5, which indicates that transcis-trans isomerization did not occur effectively to form SRG at higher temperatures.

Figure 6(a) shows the dynamics of the anomalous relaxation process after stopping laser irradiation, using a normalized relaxation parameter of the diffraction $\Delta \eta_{0}(t) . \Delta \eta_{0}(t)$ is defined by the following equation:

$$
\Delta \eta_{0}(t)=\Delta \eta(t) / \eta(1800)=[\eta(1800)-\eta(t)] / \eta(1800),
$$

where $\eta(t)$ is the diffraction efficiency at time $t$ after the irradiation of recording beam was switched off at $t=0$, which is illustrated in the inset of Fig. 6(a). In Fig. 6(a), dotted lines represent the experimental results and solid lines represent the fitted curve $[t=0$ in Fig. 6(a) corresponds to 


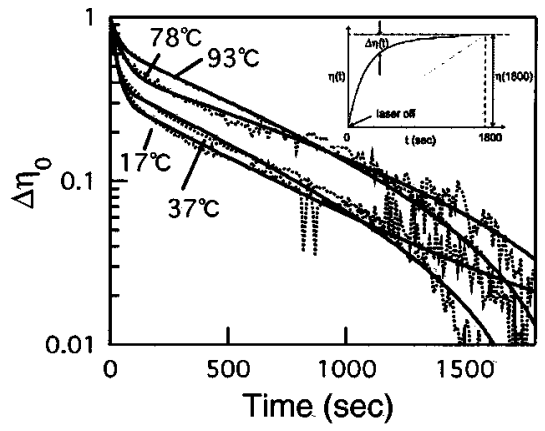

(a)

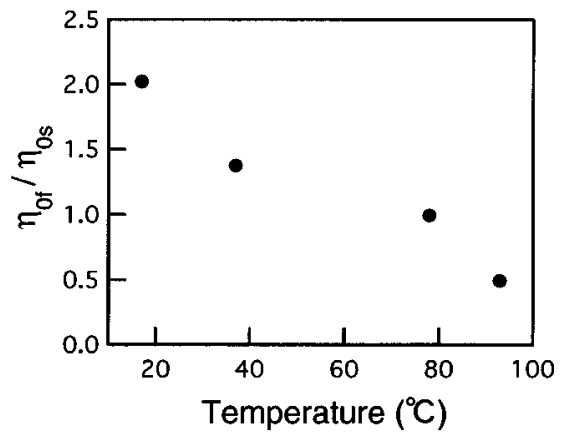

(b)

FIG. 6. (a) Decay of normalized relaxation parameters after stopping laser light irradiation at various temperatures $\left(17,37,78\right.$, and $\left.93^{\circ} \mathrm{C}\right)$. Dotted lines represent the experimental results and solid lines represent the fitted curve with double-exponential decay [time $t=0$ corresponds to that of $t=5400 \mathrm{~s}$ in Fig. 5 (a)]. Inset shows the definition of relaxation parameters of diffraction efficiency; (b) temperature dependence of the ratio of fitted coefficients $\eta_{0 f} / \eta_{0 s}$.

$t=5400 \mathrm{~s}$ in Fig. 5(a) when laser irradiation was stopped]. $\Delta \eta_{0}(t)$ can be well approximated by the double-exponential decay;

$$
\Delta \eta_{0}(t)=\eta_{0}+\eta_{0 f} \exp \left(-t / \tau_{f}\right)+\eta_{0 s} \exp \left(-t / \tau_{s}\right),
$$

where $\eta_{0 f}$ and $\eta_{0 s}$ are initial values, and $\tau_{f}$ and $\tau_{s}$ are time constants of the decay of the fast and slow components, respectively.

The double-exponential decay implies that these relaxation phenomena consist of two decay processes, that is, fast and slow decays. The fitted coefficients $\eta_{0 f}, \tau_{f}, \eta_{0 s}$, and $\tau_{s}$ are summarized in Table I. As evident from Table I, $\tau_{f}$ is approximately several tens of seconds and $\tau_{s}$ is about several hundreds of seconds. Although the time constants of the fast and slow decays are independent of temperature, the ratio of the fast and slow components strongly depends on tempera-

TABLE I. Double-exponentially fitted coefficients $\eta_{0 f}, \tau_{f}, \eta_{0 s} \tau_{s}$, and ratio of fast to slow components $\left(\eta_{0 f} / \eta_{0 s}\right)$ at various temperatures $(17,37$, 78 , and $93{ }^{\circ} \mathrm{C}$ ).

\begin{tabular}{cccccc}
\hline \hline $\begin{array}{c}T \\
\left({ }^{\circ} \mathrm{C}\right)\end{array}$ & $\eta_{0 f}$ & $\begin{array}{c}\tau_{f} \\
(\mathrm{~s})\end{array}$ & $\eta_{0 s}$ & $\begin{array}{c}\tau_{s} \\
(\mathrm{~s})\end{array}$ & $\eta_{0 f} / \eta_{0 s}$ \\
\hline 17 & 0.64 & 25.7 & 0.32 & 574.4 & 2.02 \\
37 & 0.57 & 21.9 & 0.41 & 652.7 & 1.37 \\
78 & 0.51 & 45.7 & 0.51 & 994.3 & 0.99 \\
93 & 0.34 & 32.9 & 0.70 & 737.8 & 0.49 \\
\hline \hline
\end{tabular}



(a)

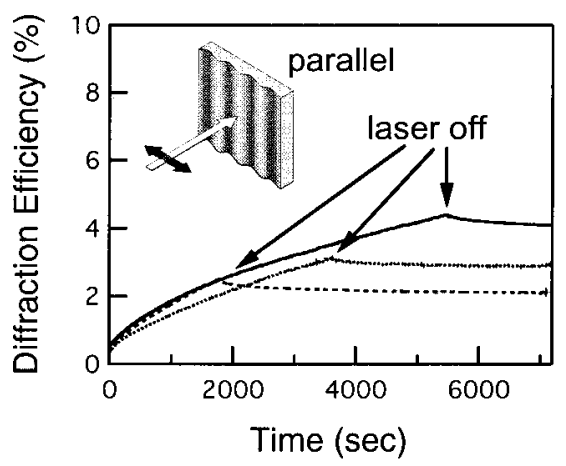

(b)

FIG. 7. Irradiation time dependence of diffraction efficiency measured with (a) perpendicularly and (b) parallel polarized probe laser beams.

ture; that is, $\eta_{0 f} / \eta_{0 s}$ is larger at lower temperatures, as shown in Fig. 6(b). This means that the contribution of the fast decay is dominant at low temperatures. However, the origin of the fast and slow decays cannot be clarified only by these results of the temperature dependence.

\section{B. Polarization effect}

As mentioned in the experimental section, the polarization of the probe $\mathrm{He}-\mathrm{Ne}$ laser beam was perpendicular to the grating vector in the previous result. However, this can be regarded to mean that the diffraction efficiency strongly depends on the polarization of the probe beam if the orientational change of azochromophores in the side chain plays a crucial role (third model). From this viewpoint, the polarization effect of the probe beam on the diffraction efficiency was investigated.

Figures 7(a) and 7(b) show the irradiation time dependence of the diffraction efficiency measured by the probe $\mathrm{He}-\mathrm{Ne}$ laser beam with the polarizations perpendicular and parallel to the grating vector of SRG, respectively. The irradiation of the $\mathrm{Ar}^{+}$laser beam for recording was performed for 30, 60, and $90 \mathrm{~min}$. For the perpendicularly polarized probe beam, the diffraction efficiency increased after the probing laser beam was switched off, as mentioned previously [Fig. 7(a)]. In contrast, when it was measured with the parallel polarized beam, the efficiency decayed as shown in Fig. 7(b). Generally, the diffraction from a Raman-Nathtype "thin" grating agrees well with the Huygens-Fresnel theory (scalar diffraction) and the efficiency change induced 


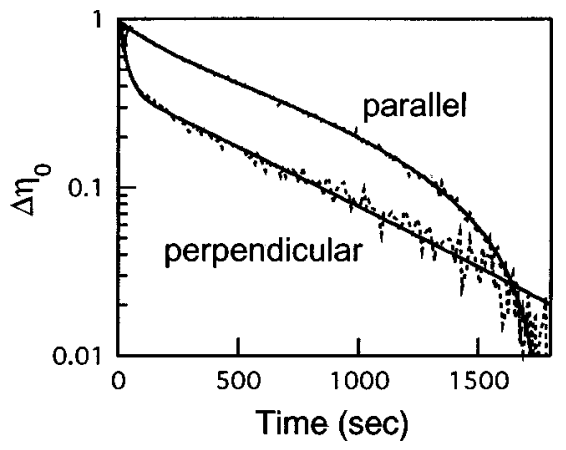

FIG. 8. Decay of normalized relaxation parameters after stopping laser light irradiation measured with perpendicularly and parallel polarized beams. Dotted lines show the experimental results and solid lines show the fitted curve with double-exponential decay.

by the change in grating profile does not depend on the polarization of the probing beam. In other words, if the diffraction efficiency of the perpendicularly polarized beam increases, that of the parallel polarized beam should also increase. Therefore, the polarization dependence of the anomalous relaxation cannot be explained by the change of the relief profile (height). From this result, the change of the relief profile (relief height) (first model) can be excluded from the explanation model of the anomalous relaxation process. As a result, it should be considered that the diffraction from "refractive index grating" plays a crucial role in the relaxation process. From Fig. 7, it was also confirmed that the anomalous relaxation process occurred independent of the irradiation time for the formation of SRG. These results ensure the validity of the speculation described above because the $Q$ factor is lower in low height grating. The difference in the diffraction efficiency measured by the light with perpendicular and parallel polarizations is remarkable also in the early stage of the formation of SRG. From this effect, the mechanism of the mass transport in the early stage may be manifested.

Figure 8 shows the time decay of the normalized relaxation parameter $\Delta \eta_{0}(t)$ after stopping the recording laser beam as a function of the polarization of the probe laser beam. In the case of parallel polarization, the decay curve can also be approximated by the double-exponential decay, as in the case of perpendicularly polarized light. From this result, it can be seen that the contribution of the fast decay is small in the case of parallel polarized light. To explain these polarization effects, the change in birefringence of the azopolymer caused by the orientational change of azochromophore in the side chain should be evaluated.

\section{Relaxation of azobenzene in the side chain}

In order to examine how azobenzene moieties reorient under the dark condition (to which direction and how large dichroism was induced), the change in absorption of azobenzene was investigated using a linearly polarized blue laser of $400 \mathrm{~nm}$ wavelength as probe light. As mentioned above, azobenzene tends to align its molecular long axis perpendicular to the polarization direction of the illuminated light through the cyclic trans-cis-trans photoisomerization

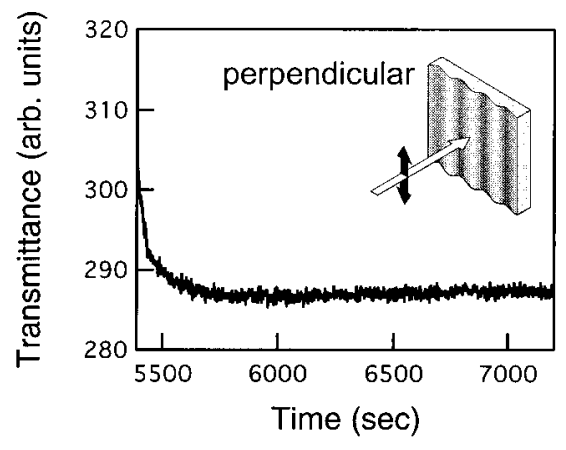

(a)

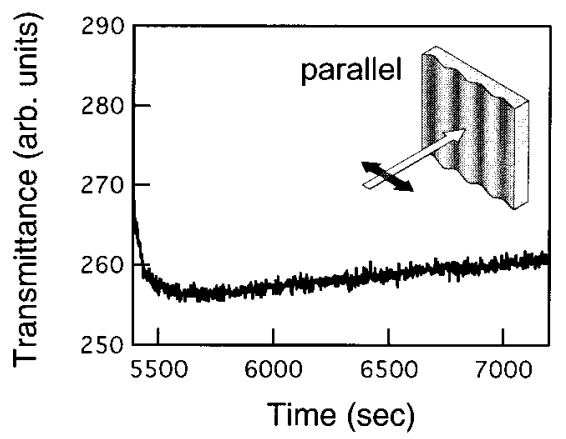

(b)

FIG. 9. Change in transmitted light intensity of probe blue laser light with the (a) perpendicularly and (b) parallel polarized light, respectively, measured after switching the recording laser light off.

(Weigert effect). To eliminate this effect, the intensity of the probe laser light was lowered. The absence of this effect was confirmed before the measurement of the change of absorption.

Figure 9 shows transmission light intensity of the probe beam with polarizations (a) perpendicular and (b) parallel to the grating vector as a function of time after the recording $\mathrm{Ar}^{+}$laser beam was switched off at $t=5400 \mathrm{~s}$. For both polarizations of the probe beam, transmission intensity decreased quickly in the early stage of the relaxation. There are two possible reasons for the transmission change upon stopping the irradiation of the recording light. One is absorption change caused by molecular reorientation. If the amount of the molecules whose transition moment is parallel to the polarization of the probe beam increases, the absorption due to $\pi-\pi^{*}$ transition of the azochromophores increases, resulting in the decrease in the transmission light intensity. If the reorientation of the azochromophores is essential, the transmission intensity of the polarized probe light parallel to the molecular long axis of azochromophores should be increased and vice versa. As shown in Fig. 8, however, the transmission intensities for both polarizations of the probe beam decrease, which cannot be explained using this model.

Another possible reason for the transmission change is the cis-trans thermal isomerization of the azochromophores. When the recording beam is switched off, the trans-cis isomerization stops and the amount of trans isomers increases, resulting in the increase in the absorption around $480 \mathrm{~nm}$; namely, the transmittance of the UV probe light 


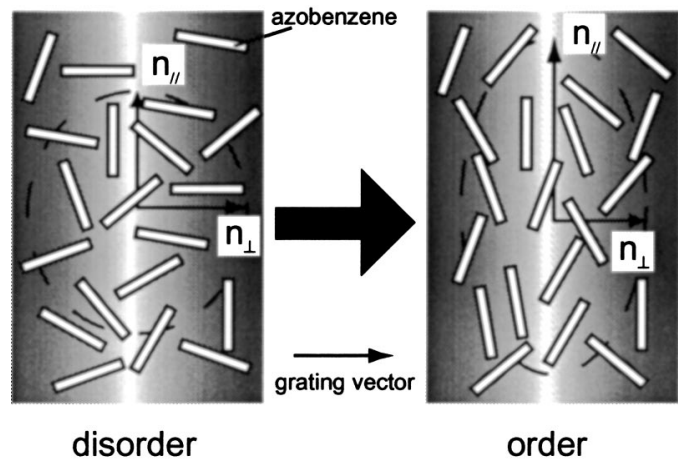

FIG. 10. Schematic explanation of disorder-to-order reorientational relaxation of azobenzene in the side chain. The ellipsoids drawn with a dashed line represent the refractive index ellipsoid of azopolymer.

(400 nm) should decrease upon stopping the irradiation of the recording light.

According to this thermal isomerization model, the temperature dependence of the efficiency change shown in Fig. 6 can also be explained; namely, the fast decay component of efficiency change was dominant at lower temperatures [Fig. 6(b)]. Because trans-cis photoisomerization did not occur effectively as shown in Fig. 5(b) at high temperatures, the amount of the cis-form of azobenzene is small. Therefore, the increment/decrement of the diffraction efficiency due to the cis-trans thermal isomerization should be small at high temperatures.

From the measurement of the dichroism and from the observation under the polarizing microscope, it was confirmed that the formed SRG had birefringence with the optical axis perpendicular to the grating vector and the averaged direction of the molecular long axis of the azochromophores aligns parallel to the groove of SRG; that is, the directional change of the optical axis after stopping the recording beam was not observed in the relaxation process. Therefore, the slow component of the efficiency change could be attributed to the orientational relaxation of each azochromophore molecule with the averaged direction of the molecular long axis remaining unchanged. In other words, the order parameter of the azochromophore in the side chain changes after the irradiation of the recording beam is stopped. As previously seen in the polarization dependence of the $\eta$ change shown in Fig. 7 , the refractive index perpendicular to the grating vector should increase and that parallel to the grating vector should decrease through the orientational relaxation.

Figure 10 shows the schematic explanation of the order parameter change of azobenzene molecules in the side chain. The ellipsoids drawn with a dashed line represent the refractive index ellipsoid of the azopolymer. As shown in this figure, with increasing order parameter of the molecular alignment, the refractive index perpendicular to the grating vector was increased and that parallel to the grating vector was decreased. This coincides with the fact that the transmittance was slightly increased after a quick decrease in the early stage, as shown in Fig. 9(b).

The driving force of this disorder-to-order orientational relaxation of the azobenzene in the side chain has not been clarified yet. However, the relaxation of azobenzene moieties should depend on the configuration of the polymer main chains and azobenzene moieties in the side chain immediately after stopping the writing laser beam; that is, the alignment of the polymer main chains, the free volume, the orientation of azobenzene moieties in the side chain, the order parameter of azobenzene moieties, etc. play important roles in the relaxation. Information on the origin of photoinduced mass transport and the formation mechanism of SRG is expected to be obtained through complete understanding of the relaxation phenomena of SRG discussed in this study.

\section{CONCLUSION}

In conclusion, the relaxation dynamics of the photoinduced SRG formed on the azopolymer film after stopping the writing laser beams has been systematically investigated. In addition to the photoinduced increase of the diffraction efficiency in the SRG, an anomalous enhancement of the efficiency was observed even without light irradiation, after the recording light beam was switched off. This anomalous relaxation process consists of two components with short and long relaxation times, and strongly depends on the temperature and polarization of the probe beam used for the diffraction measurement. At lower temperature, the anomalous growth is more effective and the fast component is dominant. From the results of the polarization dependence of the diffraction efficiency and optical absorption, it has been manifested that the fast component of the relaxation kinetics originates from the cis-trans thermal isomerization of azobenzene moieties in the side chain. The origin of the slow decay has also been discussed in terms of the order parameter change of the azomolecules caused by the reorientational relaxation. The driving force of this reorientational relaxation kinetics of the azochromophores in the side chains has not yet been clarified. However, information on the origin of photoinduced mass transport and the formation mechanism of SRG is expected to be obtained through complete understanding of the anomalous relaxation kinetics of the SRG discussed in this article.

\section{ACKNOWLEDGMENT}

This work was supported by a Grant-in-Aid for Scientific Research from the Japan Ministry of Education, Culture, Sports, Science and Technology Grant No. 13022242.

${ }^{1}$ P. Rochon, E. Batalla, and A. Natansohn, Appl. Phys. Lett. 66, 136 (1995).

${ }^{2}$ D. Y. Kim, S. K. Tripathy, L. Li, and J. Kumar, Appl. Phys. Lett. 66, 1166 (1995).

${ }^{3}$ J. Paterson, A. Natansohn, P. Rochon, C. L. Callender, and L. Robitaille, Appl. Phys. Lett. 69, 3318 (1996).

${ }^{4}$ R. J. Stockermans and P. L. Rochon, Appl. Phys. Lett. 38, 3714 (1999).

${ }^{5}$ X. T. Li, A. Natansohn, and P. Rochon, Appl. Phys. Lett. 74, 3791 (1999).

${ }^{6}$ L. Rocha, V. Dumarcher, C. Denis, P. Raimond, C. Fiorini, and J.-M. Nunzi, J. Appl. Phys. 89, 3067 (2001).

${ }^{7}$ J. Kumar, L. Li, X. L. Jiang, D.-Y. Kim, T. S. Lee, and S. Tripathy, Appl. Phys. Lett. 72, 2096 (1998)

${ }^{8}$ S. Bian, J. M. Williams, D. Y. Kim, L. Li, S. Balasubramanian, J. Kumar, and S. Tripathy, J. Appl. Phys. 86, 4498 (1999).

${ }^{9}$ T. G. Pedersen, P. M. Johansen, N. C. R. Holme, P. S. Ramanujam, and S. Hvilsted, Phys. Rev. Lett. 80, 89 (1998).

${ }^{10}$ C. J. Barrett, P. L. Rochon, and A. L. Natansohn, J. Chem. Phys. 109, 1505 (1998). 
${ }^{11}$ K. Sumaru, T. Yamanaka, T. Fukuda, and H. Matsuda, Appl. Phys. Lett. 75, 1878 (1999)

${ }^{12}$ M. Ozaki, T. Nagata, T. Matsui, K. Yoshino, and F. Kajzar, Jpn. J. Appl. Phys., Part 2 39, L614 (2000).
${ }^{13}$ T. Nagata, T. Matsui, M. Ozaki, K. Yoshino, and F. Kajzar, Synth. Met. 119, 607 (2001)

${ }^{14} \mathrm{M}$. Born and E. Wolf, Principles of Optics (Pergamon, New York, xxxx).

${ }^{15}$ R. Magnusson and T. K. Gaylord, J. Opt. Soc. Am. 68, 809 (1978). 